\title{
VII.
}

\section{Beiträge zur Pathologie der Nerven und Muskeln.}

(Eortsetzung van Bd. $127 \mathrm{S.1.}$

Vierter Beitrag.

Weobachtungen über apoplektische Alkohollahmung.

Von Prof. Dr. Hermann Eichhorst

in Zitich.

(Hierzu Tar. IV. Fig. 1 u. 2.)

Sehr bald, nachdem man sich mit der Alkoholneuritis und mit den von ihr abhängigen Lähmungen eingehender zu beschäftigen angefangen hat, ist man zu der Einsicht gekommen, dass sich dieses Leiden sehr verschieden schnell entwickeln kann. Wenn auch die Mehrzahl der Fälle einen langsamen und chronischen Verlauf erkennen lässt, so sind doch immerhin wiederholentlich Beobachtungen bekannt gegeben worden, in welchen das Krankheitsbild sowohl in Bezug auf seinen Anfang als auch in seinem Ausgang einen ausgesprochen acuten Charakter verrieth. Bernhardt') beispielsweise hat Gelegenheit genommen, in einer Arbeit, deren Beobachtungen leider der anatomischen Controle entbehren, darauf ausdriicklich hinzuweisen dass eine Gefahr besteht, eine acute Alkoholneuritis mit Landry's acuter aufsteigender Lähmung zu verwechseln. Bernhardt stützt sich dabei auf zwei Mittheilungen von Broadbent ${ }^{2}$ ) und Myrtle ${ }^{3}$ ), von welchen wir freilich bemerken müssen, dass ihre Unter-

9) R. Bernhardt, Ueber multiple Neuritis der Alkoholisten. Beiträge zur differentiellen Diagnostil dieses Leidens von der Tabes, der Poliomyelitis subacuta und der sog. Landry'schen Paralyse. Zeitschrift f. klin. Med. Bd. XI. 1886. S. 362 .

3) W. H. Broadbent, On a form of alcoholic spinal paralysis. Medic. Chirurg. Transact. Vol. 67, 1884. p. 133.

3) Myrtle, On a case of acute ascending paralysis, chronic alcoholism. Brit. Med. Journal. 1882. Aug. 19. p. 312. 

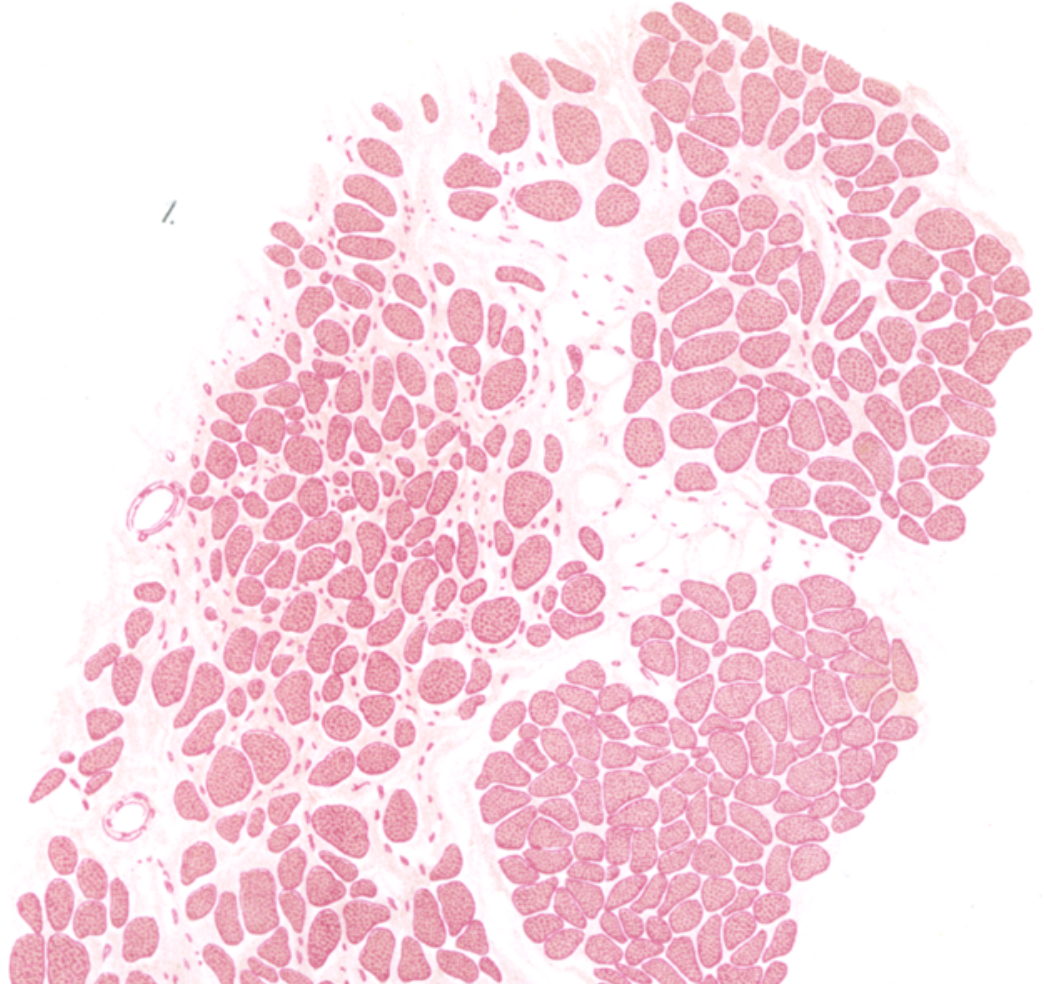

U 05.9503

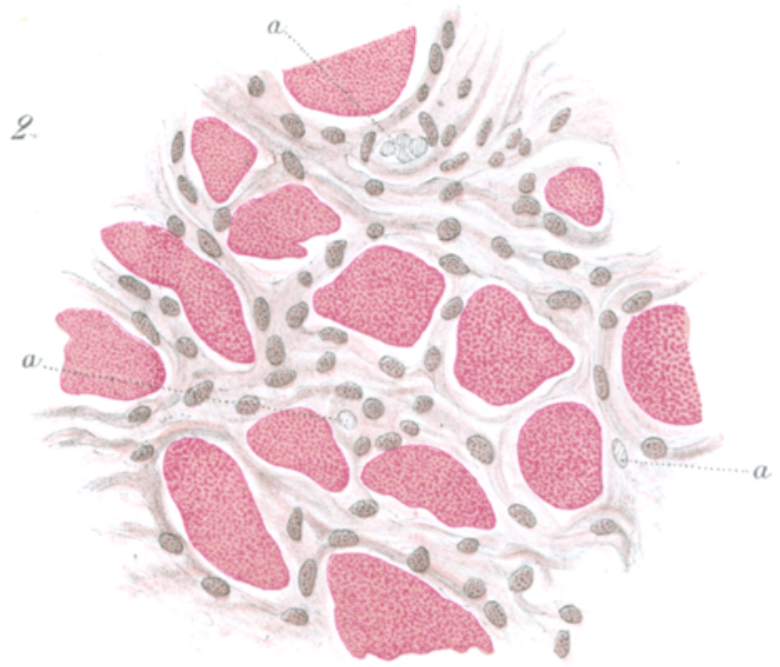

Alb. Sohinze Phromolith Berlin 
suchung viel zu wünschen übrig lässt, um als einigermaassen erschöpfend angesehen werden zu können.

Von einem apoplektischen Einsetzen einer Alkohollähmung ist mir. in der einschlägigen Literatur Nichts bekannt geworden. Dass Neuritiden anderen Ursprunges urplötzlich mit Lähmungen beginnen können, dafür sind vereinzelte Beispiele beschrieben worden. Als ich in diesem Archiv ${ }^{1}$ ) im Jahre 1877 den allerersten Fall von acuter Polyneuritis bekannt machte, handelte es sich um Lähmungen, welche einen sehr ausgeprägten apoplektischen Charakter besassen, und bei der mikroskopischen Untersuchung der erkrankten Nerven gelang es mir, Blatungen innerhalb des Endoneuriums nachzuweisen. Erst vor kurzer Zeit haben Déjérine ${ }^{2}$ ) and Dubois ${ }^{3}$ ) ähnliche Erfahrungen mitgetheilt.

Dass auch die Alkohollähmung einen apoplektischen Anfang darbieten kann, soll im Folgenden an einigen Beispielen aus meinem Erfahrungskreise nachgewiesen werden.

Nach dem, was man bis jetzt über peripherische apoplektische Lähmungen und über apoplektische Neuritis wusste, hätte man erwarten sollen, dass auch bei der apoplektischen Alkohollähmung Blutungen in den peripherischen Nerven den Grund abgeben. Dieser Gedanke lag um so näher, als Wernicke ${ }^{4}$ und Thomsen ${ }^{5}$ ) bei der alkoholischen Ophthalmoplegie Blutungen in den Kernen der Augennerven nachgewiesen haben. Allein unsere mitzutheilenden Beobachtungen lehren, dass es Alkohollähmungen von apoplektischem Charakter giebt, welche mit Blutungen Nichts zu thun haben, denen überhaupt nur functionelle Störungen zu Grunde liegen, Lähmungen, welche bald peripherische, bald centrale Eigenschaften darbieten, - und schon aus diesem Grunde dürften dieselben einer genaueren Beschreibung nicht unwerth erscheinen.

1) H. Eichhorst, Neuritis acuta progressiva. Dieses Archiv Bd. 69. 1877. S. 265.

2) Déjérine, Sur un cas de neurite apoplectiforme du plexus brachiale suivi d'autopsie. Compt. rend. de la société de biologie. 1890. No. 27.

3) Dubois, Correspondenzbl. f. Schweizer Aerzte. 1890. S. 9.

1) C. Wernicke, Lehrbuch der Gehirnkrankheiten. Bd. II. S. 235 u. 236.

5) R. Thomsen, Zur Pathologie und Anatomie der „acuten“ alkoholischen Augenmuskellähmungen. Arch. f. Psychiatr. Bd.19. 1886. S. 185. 
Beobachtuag I.

Win 37 jähriger Buchhalter, welcher notoriseher Säufer ist, kommt wegen allgemeiner Fettsucht und Lebereirhose zur Aufnabme anf die medicinische Klinik. Er wird hier fast vier Wochen lang beobachtet. Ungefähr 14 Tage vor seinem Tode stellen sich Delirien ein. 3 Tage vor dem Tode treten plötzlich Lähmungen der vom Radialis innervirten Vorderarmmusculatur ein. Tod unter zunehmenden Delirien.

Anamnese. Georg Nätholz, 37 Jahre alt, Buchhaiter aus Appenzell, aufigenommen am 26. Angust 1889, gestorben am 23. September 1889.

Der Vater des Patienten starb vor wenigen Monaten an einem Nierenleiden: er war sebr fettleibig und hatte Zucker im Harn. Die Mutter starb an Gesichtsrose.

Als Kind hat Patient Masern und Pocken durchgemacht. Während der Jahre $1880-1885$ hielt er sich in Amerika anf und uberstand hier eine Krankheit von vierwöchentlicher Dauer, über welche er nichts Anderes au berichten weiss, als dass die Beine geschwollen waren. Vielleicht babe es sich, so meint Patient, um Rheumatismus gehandelt. 1887 wurde er von Nervenfieber befallen. Im Anschluss daran stelle sich Schwellung im linken Bein ein, um deretwillen Patient längere Zeit das Bett hüten musste. Im Verlauf dieser Veränderung schuppte sich am linken Bein die Haut sehr lebhaft.

Der Kranke war ein starker Esser und sprach dem Alkohol in jeder Form sehr wacker $z u$. Als er 1885 ans Amerika zurückkehrte, begann er sehr fett zu werden. Seit drei Monaten nahw die Fettleibigkeüt ganz besonders zu, so dass sein Körpergewicht um 20 Pfund wuchs. Er hatte zunächst bis auf Mattigkeit keine besonderen Beschwerden. Seit 14 Tagen traten Schmerzen im Bauchraum auf, welche namentich nach den Mahlzeiten beträchtlich wurden. In den letzten Tagen nahm das Gefübl der Entkräfung so zu, dass sich Patient auf die medicinische Klinik aufnehmen liess.

Status praesens 27. August 1891. Mittelgrosser Mann von kräftigem Knochenbau, schlaffen Muskeln, aber mit einem ungewöhnlich reichlich entwickelten Fetipolster. Die Hautdecken lassen eine schwache, aber deutliche icterische Verfärbung erkennen, und dasselbe gilt auch für die Conjunctiva sclerarum. Dabei ist die Haut trocken, aber geschmeidig, fast fettig anzufüblen.

Patient nimmt active Räckenlage ein und vermeidet gern Seitenlage, da er dabei kurzathmig werde. Das Bewusstsein ist volkommen klar. Die subjectiven Beschwerden äussern sich in Karzathmigkeit, grosser Mattigkeit und Beengung und Schmerzen im Bauchraum.

Achsehhöhlentemperatur $36,8^{\circ} \mathrm{C}$, Puls regelmässig, klein, wenig gespannt und beschleunigt, 104 Schläge binnen einer Minute. Athmung beschleunigt (32 Athmungszüge in einer Minute), sehr fiach und rorwiegend abdominal. Keine Oedeme. 
Gesichtszüge plump und ausdruckslos. Gesichtsfarbe blass und leicht icterisch. Pupillen von mittlerer Weite, beiderseits gleich und auf Lichteinfall trefflich reagirend. Nasenwurzel etwas eingesunken und über der Einsenkung eine alte Hautnarbe sichtbar. Zunge feucht, auf der Oberfläche mit einem dünnen grauen Belage bedeckt. Beim Oeffnen des Mundes macht sich auf der Schleimbaut der Wangen und des harten Gaumens sehr deutlich eine icterische Verfärbung bemerkbar. Mandeln etwas vergrössert. Die hintere Rachenwand sebr lebhaft geröthet. Beim Versuch, den Mundspatel auf den Zungenrücken zu legen, treten sehr heftige Würgbewegungen auf und offenbart sich eine ungewöhnliche Ueberempfindlichkeit.

Der Hals ist sehr kurz. Es besteht eine rechtsseitige Struma, etwa von der Grösse eines kleinen Apfels.

Thorax breit, kurz und von einem gewaltigen Fettpolster umgeben, durch welches man die Intercostalräume und Rippen nur sehr schwer und undentlich abtasten kann. Es besteht vorne beiderseits bis zur dritten Rippe lauter, nicht tympanitischer und gleicher Lungenschall. Rechts wird der Percnssionsschall bereits unterbalb der dritten Rippe sehr deutlich gedämpft und von der vierten Rippe an besteht Schenkelsehall. Die Dämpfungsgrenze lässt geringe respiratorische Verschiebungen erkennen. Soweit keine Dämpfung besteht, hört man vesiculäres Athmungsgeräusch, welches am Anfang der Dämpfung leise wird, um sehr schnell ganz zu rerschwinden.

In der rechten Thoraxseite findet man rechts bis zur vierten Rippe lauten Percussionsschall und vesiculäres Athmungsgeräusch, links bis zar sechsten Rippe.

Hinten besteht bis zum unteren Schulterblattwinkel beiderseits gleicher, lauter, nicht tympanitischer Lungenschall, dann beginnt rechts eine leichte Dämpfung, über welcher das vesiculäre Athmungsgeräusch und der Stimmfremitus abgescbwächt sind. Ueber den nicht gedämpften Abschnitten der hinteren Thoraxfläche vernimmt man äberall Vesiculärathmen.

Patient bat sehr wenig rein schleimigen Auswurf ansgehustet.

Der Spitzenstoss des Herzens kann weder gesehen noch gefühlt werden und auch über den oberen Herzabschnitten ist niebts von den Herzbewegungen wahrnebmbar. Die Grenzen der grossen Herzdämpfung bewegen sich zwischen dem oberen Rande der dritten linken Rippe, dem oberen Rande der secbsten linken Rippe, der linken Mamillarlinie und linken Sternallinie. Die Herztöne sind sehr dumpf, doch rein.

Das Abdomen fällt durch seine gewaltige Wölbung auf und erreicbt in der Höhe des Nabels einen grössten Umfang von $129 \mathrm{~cm}$. Die Entfernung vom Schwertfortsatz bis zum Nabel beträgt 30 und diejenige zwischen Nabel und Symphysis pubis $21 \mathrm{~cm}$. Uebrigens erscheint die rechte Baucbseite etwas lebbafter gewölbt als die linke. Die epigastrische Gegend springt fast balbkuglig nach rorn hervor.

Der rechte Bauchraum und das Epigastrium sind von einem umfangreichen und harten Körper erfüllt, welcher nach unten bis an das rechte Ligamentum Poupartii reicht, in der yerlängerten rechten Parasternallinie 
144

ziemlich senkrecht mit seinem medianen Rande nach oben steigt und dan $18 \mathrm{~cm}$ unter dem Schwertfortsatze und $14 \mathrm{~cm}$ oberhalb des Nabels anfang: nabezu horizontal, dann nach links obea läuft, um in der linken Mamillarlini, binter dem linken Brustkorbrande zu verschwinden (vergl. untenstehende $A b$ bildung). Etwa $11 \mathrm{~cm}$ rechts vom Nabel entfernt lässt sich an der beschrie benen Masse eine deutliche Einkerbung erkennen. Nirgends machen sich äbe

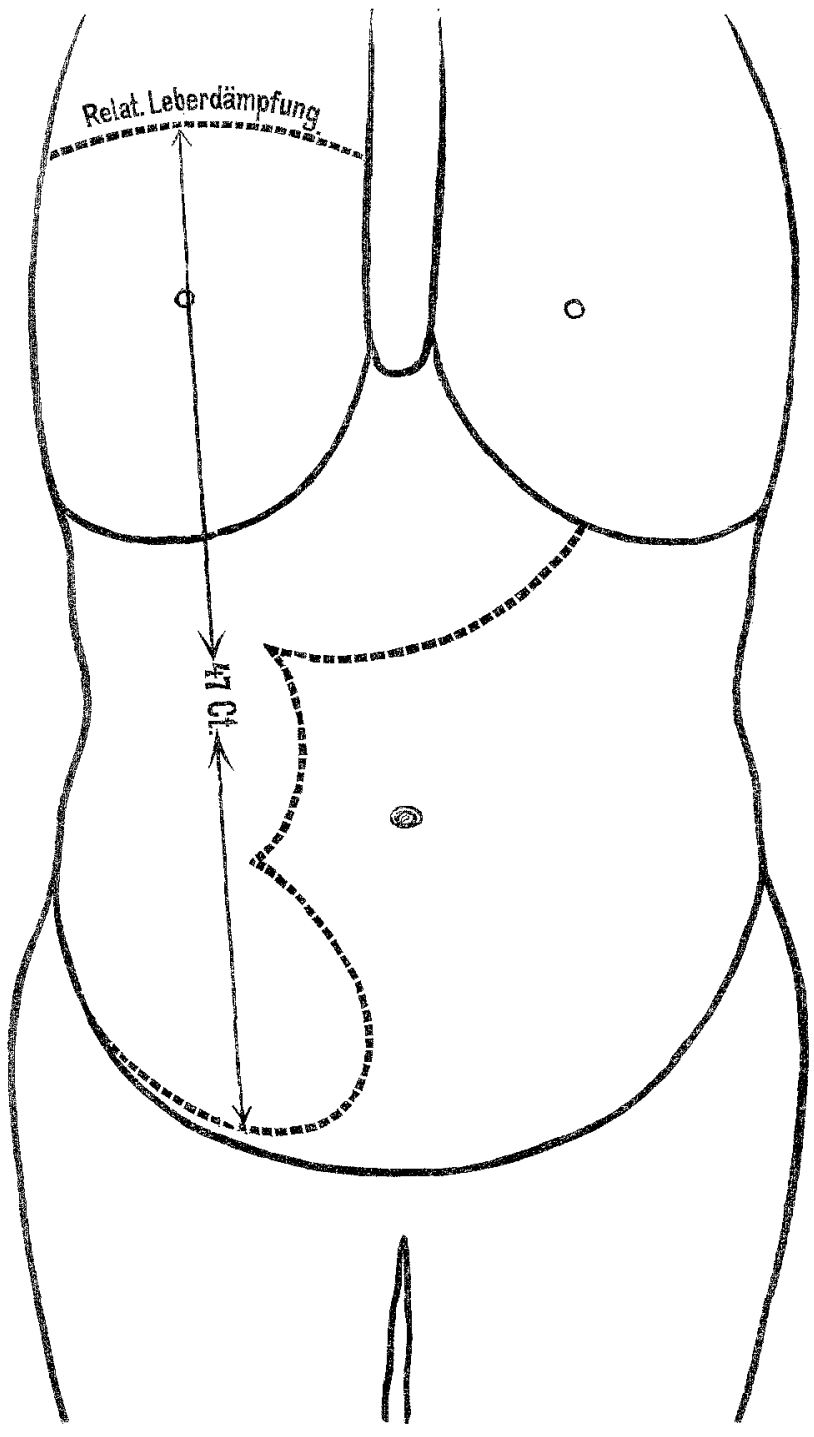


dem Gebilde Hervorragungen bemerkbar und nur eine kleine Stelle im Bereiche der Mittellinie ist gegen stärkeren Druck etwas empfindlich. Der Rand ist überall scharfkantig und deutlich abtastbar, das ganze Gebilde allerorts von sebr harter Consistenz. Nach oben gebt die Dämpfung unmittelbar in die Leberdämpfung über; auch lässt sich an dem linken Rande eine zwar geringe, deunoch aber dentliche respiratorische Verschiebung nachweisen. Die Entfernung zwischen dem Beginn der oberen Leberdämpfung am unteren Rande der dritten rechten Rippe und dem untersten Rande des Tumors beträgt $47 \mathrm{~cm}$.

Die Milz ist in rechter Diagonalstellung deutlich fühlbar und sehr hart. Oben beginnt die Milzdämpfung an der achten linken Rippe und unten endet sie am linken Brustkorbrande. Vorn überragt sie ein wenig den Brustkorbrand.

Sonst giebt das Abdomen überall tympanitischen Schall und erweist sich gegen Druck nirgends empfindlich oder resistent.

Auf der Haut der Untersehenkel bemerkt man einzelne braunrothe Flecken, die von alten Blutpigmentresten herrühren. Druck auf die Tibien etwas empfindlich, auch das Brustbein etwas druckempfindlich, dagegen sind die Arme schmerzfrei und von sehr bedeutender Kraft.

Patient hat wäbrend der letzten 12 Stunden $800 \mathrm{ccm}$ Harnes entleert. Der Harn ist dunkelroth, giebt aber weder mit Salpetersäure noch mit Jodtinctur Gallenfarbstoffreaction, enthält kein Eiweiss und setzt ein Sediment von Uraten ab. Sein specifisches Gewicht beträgt 1024. Der Kranke hat täglich 1-2 feste Stühle, bat keinen Appetit, dagegen viel Durst und schläft während der Nacht unruhig und mit vielfachen Unterbrechungen.

Bei der Untersuchung des Blutes findet man nichts Abnormes, namentlich beine Poikilocytose und Vermehrung der farblosen Blutkörperchen.

Ordo: 1) Flüssige Diät, namentlich Mileh.

2) Sol. Kalii jodat. $5,0: 200$.

3 mal täglich 1 Esslöffel.

Krankbeitsverlauf.

Die Körpertemperatur des Patienten verbielt sich fast inmer normal und bewegte sich zwischen $36,4-37,4^{\circ} \mathrm{C}$. Nur an 11 . September erreichte sie am Abend $37,2^{\circ} \mathrm{C}$. und am 13. September $37,8^{\circ} \mathrm{C}$. Patient hatte beim Eintritt des Todes $37,3^{\circ}$ und zeigte 15 Minuten nach dem Tode $38,5^{\circ} \mathrm{C}$. Der Puls war meist beschleunigt und schwankte zwischen 96 und 124 Schlägen. Die Diurese hielt sich innerhalb normaler Werthe $(1200-1800 \mathrm{ccm})$. Der Harn behielt eine saturirte Farbe, enthielt reichlich Urate, blieb aber eiweissfrei und wechselte mit seinem specifischen Gewicht zwischen 1015 bis 1026. Patient klagte in der Regel äber Enge und Bangigkeit, zeitweise auch über Schmerzen in den Extremitäten, namentlich in der Nähe der grossen Gelenke, ohne dass man an diesen irgend welche Veränderungen ausfindig machen konnte.

Am 11. September fiel es auf, dass der Hauticterus dentlich zugenommen batte. Der Patient schien ausserdem verwirt. Seit diesem Tage treten. 
namentich wabrend der Nacht lebhaftere Delirien ain, aber auch am Tuge ist Patient nicht bei klarem Bew usstsein.

Aw 21. September baben sich während der Zeit von 4 Uar bis 6 Uhr Morgens plötzlich Labmungen der Streckunculatur dej Vorderar me ausgebildet. Patient erscheint an Morgen soweit bei Besinnung, dass er die ärztliche Aufforderung versteht, die volarwärts flectirten. Hände dorsalwärts zu erheben, die gebeugten Finger zu strecken und den an die Hände angezogenen Daumen zu abduciren, aber er ist nicht im Stande, dem Geheisse nachzukommen. Eine Streckung der gebeugten Unterarme gelingt. Schwere Sensibilitätsstörungen bestehen jedenfalls nicht. Die Lähmung dauert an den nächsten beiden Tagen in unveränderter Weise fort. Die Prüfung der geläbmten Muskeln mit dem faradisehen Strom ergiebr nichts abnormes. Am 23. September fallt die grosse Kleiaheit und Schwäche des Pulses auf. Gegen Mittag verfällt Patient in tiefe Delirien; um $9 \frac{1}{2}$ Ubr Abends tritt ziemlich plötzlich Trachealrasseln auf und rach einer halben Stunde ist der Kranke verstorhen.

Section am 24. Septembe 1889. Kräftige, grosse männliche Leicbe von gutew Ernäbrungszustand und mit zahlreichen Todtenflecken auf den hinteren Partien des Körpers. Hautfarbe gelblich und deutlich ieterisch.

Bei Herausnahme des Rückenmarkes zeigen sich die verösen Plexus des Wirbelkanales stark mit Blut gefüllt. Die Dura mater spinalis erscheint auf der äusseren und inneren Fläche unverändert. Das Rückenmark ist von guter Consistenz, seine weisse Substanz blutarm, die Zeichnung der grauen Substanz sebr deutlich. Mikroskopisch lassen sich im Rückenmarke keine Veränderungen erkennen.

A uf der Rückenbant fält die enorme Entwickeluug des Fettpolsters auf. Anch bei Herausnahme der beiden Ischiadici findet man die Glutealmusculatur von sebr reichlicben Fettwassen durchsetzt. Uebrigens bieten die Querschnitte der Ischiadici keine Auffälligkeiten dar. Auch an den U1nar- und Radialnerven jst nichts Ungewöbnliches zu sehen.

Gesicht stark cyanotisch. Schädel lang und mit gröstentheils gesehwundener Diploë. Schädelnähte kaum noch erkennbar. Dura mater mässig gespannt. Ix Sinus longitudinatis wenig flüssiges Blut. Innenfäche der Dura mater glatt. Pia mater vielfach verdickt. Ihre Gefässe stark wit Blut gefüllt. An den basalen Hirnarterien keine nennenswerthe Veränderung. Seitenventrikel und dritter Hirnventrikel ziemlich stark erweitert. Die Hirnsubstanz ist im Aligemeinen blutreich und ron schlaffer Consistenz. Auch die basalen Grosshiraganglien erscheinen blutreich, sonst aber ohne Yeranderung. Ebenso wenig kann man am Boden des vieten Ventrikels etwas Auffälliges finden und im Besonderen erscheint sein Ependym nicht granulirt.

Bauch stark aufgetrieben. Fet tpolster colossal entwickelt. Musculasur braunroth, schlaff und überall von Fettmassen durchsetz. Netz sebr fettreich; das Fett ron citronengelber Farbe. Därme wässig injicirt und theil weise stark durch Gas aufgetrieben. Die Leber durch die Därme in die Höhe gedrängt, so dass sie nur mit dem unteren Rande sichtbar ist. 
Rippenknorpel grösstentheils stark verkalkt. Aus den grossen Halsvenenstämmen ergiesst sich sehr viel Blut.

Linke Pleurahöble leer und linke Lunge frei. Rechte Lunge au mehreren Stellen verwachsen, namentlich im Bereiche des Unterlappens.

Herzbeutel aussen sehr fettreich; ebenso sehr viel Fett unter dem Epicard. Herzbeutel leer. Herz sehr gross und schlaff. Herzgewicht $560 \mathrm{~g}$. Im rechten Herzen viel fiüssiges Blut. Rechter Ventrikel und Vorhof stark erweitert. An den Pulmonal- und Tricuspidalklappen, welche zart sind, nichts Besonderes. Die Musculatur blass und schlaff. Die Papillarmuskeln sind mit vielfachen gelben Fleckehen durchsetzt. Unter dem Epicard des rechten Ventrikels mehrere Blutungen. Linker Ventrikel stark erweitert; sein Endocard blutig imbibirt, ebenso die Aortenintima und das Endocard des linken Vorhofes. An den linksseitigen Herzklappen keine Besonderbeiten. Musculatur des linken Ventrikels sehr schlaff, blass, von gelben Flecken durchsetzt, welche letzteren sich besonders reichlich in den Papillarmuskeln zeigen.

Linke Lu nge im Allgemeinen ziemlich gut lufthaltig. Ihr Unterlappen stark bluthaltig. Der Oberlappen stark ödematös, namentlicb gegen die Spitze hin, während der untere Theil luftleer und von grauröthlicher Farbe ist, auf Druck träbes Fluidum ausdrücken lässt und leicht einbricht. Bronchialscbleimhant stark geröthet und mit schaumiger Flüssigkeit bedeckt.

Rechts findet man unter der Pleura des Unterlappens einige Blutungen und erophysematöse Stellen. Der Unterlappen sehr blutreich. Im Mittellappen eine alte schieferige Narbe, sonst keine Verānderung. Oberlappen stark ödematös. Bronchialschleimbaut wie links.

Einzelne Bronchialdrüsen stark pigmentirt und leicht verkalkt.

Milz stark vergrössert und ihre Kapsel mit vielen fibrösen Verdickungen bedeckt. Milzgewicht $676 \mathrm{~g}$. Milzmaasse: 19,14 und $5 \mathrm{~cm}$. Auf dem Durchschnitte treten die Follikel besonders deutlich hervor. Pulpa bellröthlich und schlaff.

Linke Nebenniere klein, mit stark verfetteter Rinden- und dunkel gerötheter Marksubstanz. Fettkapsel der linken Niere colossal fettreich. Niere gross. Nierenkapsel leicht abziehbar. Rinde auf der OberfÄäche und auf dem Nierendurchschnitt von gleichmässig dunkelblaurother Farbe. Auf der Nierenoberfläche sind einzelne Cysten und verkalkte Glomeruli bemerzbar. Das Gewicht der linken Niere ohne Kapsel beträgt $250 \mathrm{~g}$.

Rechte Nebenniere und rechte Niere wie links. Auf der Nierenoberfläche findet sich ein kleiner älterer Infarkt. Ausserdem zeigt sich starke Verkalkung einzelner Pyramidenspitzen. Nierengewicht $260 \mathrm{~g}$.

Ductus choledochus durchgangig. Auf der Scbleimbant des Magens und Duodenums findet man ziewlich viele Blutungen.

Die Leber ist namentlich im rechtẹn Lappen colossal vergrössert, im linken Lappen dagegen eher verkleinert. Die Oberfläche zeigt besonders über dem linken Lappen eine grosse Menge von Ungleichheiten und knotenförwigen Hervorragungen. Die Glisson'sche Kapsel ist nirgends auffälig 
verdiclat oder getrubt. Die Leber ist ziemulich blutreich and von lebbat icterischer Farbe. Die einzelnen Leberzellen zeigen sich durch grössere und breitere Bindegewebszüge von einander getrennt. Die Farbe der einzelnen Inseln ist gelblich-grün. Eine dentiche Fettrerfärbung ist wegen zu staiken leterus nicht zu constatiren.

Blase und Geschlechtsorgane bieten nichts Besonderes dar.

Im rechten Schilddrüsenlappen starke Hyperplasie, in linken eine grosse Cyste.

In der Aorta nur leichte Verfetungen, keine Arteriosklerose.

Anatomische Diagnose. Allgemeine Fettacht. Hypertrophische Lebercirrhose. Chronischer Milatumor. Pneumonie im linken Oberlappen. Struma.

Von Leichentheilen waren behufs mikroskopischer Untersuchung ausser der Leber noch das Rückenmark, die Ischiadici, die Radial- and Ulnarnerven, sowie Stücke des Muscalas extensor digitorum der Unterarme und des Biceps der Oberschenkel aufgehoben worden.

Leberstückchen wurden in Alkohol erhärtet und dann an Wikrotomschnitten vornehmlich mit Hämatoxylin oder mit Hämatoxylin und Eosin gefärbt. Die Leber zeigte zunächst das Bild einer sebr hochgradigen interstitiellen Bindegewebswucherung. Die Leberläppchen waren durch sehr breite Züge von Bindegewebe von einander getrennt und vielfach bis auf kleine Leberzellenreste geschwunden. Während das gewucherte Bindegewebe in den mittleren Partien eine schwielige und zellenarme Beschaffenheit darbot, zeigten sich die den Leberläppchen zunächst gelegenen Abschnitte wesentlich zellenreicher. Vor Allem aber fiel hier eine sehr lebhafte Wucherung der Gallengänge auf, welche ein weitverzweigtes und vielfach mit einander anastomosirendes Netz bildeten. Dabei zeichneten sich die Epithelzellen in den Gallengänges durch eine beträchtliche Grösse aus. Nicht selten beobachtet man in dem neugebildeten Bindegewebe Blutgefässe, welche durch Wucherungsvorgänge auf der Gefässintima entweder versehlossen waren oder einem völligen Verschluss sehr nahe standen. An anderen Stellen dagegen schienen sich neue Blutbahnen eröfnet zu haben; man sah hier weit klaffende Gefässlichtungen, mit einer sehr dünnen kernreichen Wand. Hier und da waren noch in dem Bindegewebe Körnchen, Kleckse und Klumpen von Gallenpigment bemerkbar. Hervorgehoben mag noch werden, dass stellenweise in den Le- 
berzellen eine sebr bedeutende Verfettung Platz gegriffen hatte, in der Regel freilich derart, dass in einem Leberläppchen fast sämmtliche Leberzellen hochgradig verfettet waren, während sich in vielen anderen nicht einmal Spuren von Verfettung nachweisen liessen.

Rückenmark, Nerven und Muskeln wurden zunächst in Müller'scher Flüssigkeit bis zur Schnittreife gehärtet. Mikrotomschnitte des Rückenmarkes wurden vornebmlich einer Behandlung mit essigsaurem Carmin und Salzsäure-Glycerin unterzogen. Das Rückenmark zeigte sich als in jeder Beziehung unversehrt und auch an den Wurzeln des Rückenmarkes liess sich keine Veränderung erkennen.

Mit einer gewissen Spannung trat man an die Untersuchung der peripherischen Nerven heran; aber auch an ihnen war man nicht im Stande eine krankhafte Veränderung wahrzunehmen. Feine Querschnitte der Radial- and Ulnarnerven waren den verschiedensten Färbungsmethoden (Lithioncarmin, Hämatoxylin, Eosin-Hämatoxylin u. s. f.) unterzogen worden, sie zeigten sich als unverändert. Die Nervenfasern waren fast durchgehends von grossem Kaliber und feine Nervenfasern fanden sich nur in geringer and vereinzelter Zahl. Aus dem mikroskopischen Bilde war man nicht im Stande zu entscheiden, ob man den Querschnitt des Ulnar- oder Radialnerven vor sich habe. Es hatte sich also bei der anatomischen Untersuchung des Nervensystemes eine Aufklärung über Natur und Sitz der apoplektischen Radialislähmung nicht gefunden.

Fast schien es, dass die mikroskopische Untersuchung der Muskeln den gewünschten Aufschluss bringen sollte, denn ohne allen $\mathrm{Zweifel} \mathrm{liessen} \mathrm{sich} \mathrm{hier} \mathrm{nicht} \mathrm{unbedeutende} \mathrm{Veränderun-}$ gen nachweisen. Auf Muskelquerschnitten zunächst der Fingerextensoren fiel es auf, dass das Perimysium internum überall sehr kernreich war. Ausserdem machte sich aber an vielen Orten eine sehr ausgebildete Wucherung des intrafasciculären Bindegewebes bemerkbar, so dass die einzelnen Muskelfasern durch ungewöhnlich breite Räume von einander getrennt waren (vgl. Fig. 1). Das gewucherte Bindegewebe war sehr zellenreich und zeichnete sich durch einen welligen, lockigen Bau aus (vgl. Fig. 2). An vielen Stellen sah man in seiner Mitte 
stapk gefillte Blutcapillaren liegen, and es wurde mehrfach der Eindruck wachgernfen, als ob die Vermehrung des Bindegewebes von der Aussenwand der. Capillaren ihren Anfang genommen habe. Auf die anliegenden Muskelfasern war die Wucherung des Bindegewebes nicht immer ohne Einfluss geblieben, und es kamen an zahlreichen Stellen Bilder zum Vorschein, welche in unzweidentigster Weise für einen allmählichen Schwund der Muskelfasern sprachen. Traf man doch hier und da nur noch schmale Reste von Muskelfasern an.

Hätte man die Untersuchung der Muskeln auf die Fingerstrecker beschränkt, so wäre man wohl zu dem Schlusse gelangt, dass die während des Lebens beobachtete Radialislähmung wahrscheinlich myogenen Ursprunges sei. Ist doch neuerdings wiederholt und zuerst und am eingehendsten von Senator') darauf hingewiesen worden, dass sehr innige Beziehungen zwischen Myositis und Neuritis zu bestehen scheinen, so dass eine Myositis erst nachträglich zu einer Neuritis führen könne. Vielleicht lag auch in unserer Beobachtung eine Myositis vor, bei welcher es zur Ausbildung einer Neuritis nicht kommen konnte, weil der Tod aus anderen Ursachen zu früh eintrat, denn dass der Alkohol ausser den peripherischen Nerven auch das Muskelgewebe zu schädigen im Stande ist, haben zur Genüge Erfahrungen, die bereits früher von mir in diesem Archive ${ }^{2}$ ) mitgetheilt wurden, sowie auch Untersuchungen von Siemerling ${ }^{3}$ ), Thomsen ${ }^{4}$ ) and Oppenheim ${ }^{5}$ ) ergeben. Es darf freilich nicht übersehen werden, dass zwar eine ausgebreitete Myositis die Lähmung, aber wohl kaum den apoplektischen Charakter der Lähmung erklärt hätte. Wir mussten aber von einer myogenen Auffassung der Lähmung überhaupt zurückkommen, als es sich bei der mikroskopischen Untersuchung des Biceps femoris herausstellte,

1) H. Senator, Ueber acute und subacute multiple Neuritis und Myositis. Zeitschr. f. klin. Mled. Bd.XV. 1889. S.61.

$\Rightarrow$ H. Eichhorst, Neuritis fascians. Dieses Archir Bd. 112. S. 237. 1888.

3) Siemerling, Ein Fall ron Alkoholueuritis mit hervorragender Betheiligung des Muskelapparates u. s. w. Charité-Annalen 1889. S.443.

4) R. Thomsen, Zur Klinik und patbologischen Anatomie der multiplen Alkoholneuritis. Arch. f. Psychiatr. Bd.XXI. 1890. \$.806.

5) H. Oppenbeim, Weitere Mittbeilungen zur Pathologie der multiplen Neuritis. Berl. klin. Wochenschr. 1890. No. 24. \$.545. 
dass hier genau die gleichen Veränderungen und zwar in der gleichen Ausdehnung anzutreffen waren. Da während des Lebens Lähmungen an den unteren Extremitäten nicht bestanden hatten, so musste man daraus folgern, dass sich sowohl an den Armen wie auch an den Beinen die Mukelveränderungen ohne greifbare functionelle Störungen vollzogen haben mussten. Vielleicht, dass es über kurz oder lang zu myogenen Lähmungen gekommen wäre. Nicht unmöglich will es mir erscheinen, dass die sehr lebhaften Schmerzen in den Extremitäten, welche der Kranke vorwiegend in die Nähe der grossen Gelenke verlegt hatte, mit den Muskelveränderungen in Zusammenhang stehen könnten.

Wenn sich nun für die apoplektische Radialislähmung weder an den Nerven noch an den Muskeln eine greifbare Veränderung nachweisen liess, wie sollte man sich ibr Zustandekommen erklären? Nach meinem Dafürhalten handelte es sich um eine rein functionelle Lähmung. Ich verkenne keinen Augenblick, dass man dabei mit einem Factor rechnet, welcher ausserordentlich gefährlich werden und missbraucht allem Unbegreiflichen eine Art von Scheinerklärung verleihen kann. Allein sind denn anf dem Gebiete toxischer Einflüsse des Nervensystemes functionelle Störungen ohne anatomische Grundlage etwas so Ungewöhnliches? Es kann doch keinem gerechten Bedenken begegnen, dass beispielsweise das Blei im Stande ist, in der allerschwersten Weise die Thätigkeit des Gehirnes zu beeinträchtigen, ohne dass es bisher möglich gewesen ist, anatomische Veränderungen dafür als Grund nachzuweisen. In meinen letzten Beiträgen zur Pathologie der Nerven und Muskeln ${ }^{1}$ ) habe ich gezeigt, dass das Verschwinden des Patellarsehnenreflexes bei Zuckerharnruhr bald mit neuritischen Veränderungen zusammenhängen, bald aber reiner functioneller Natur sein kann. Ueber functionelle Störungen des Nervensystemes durch Alkoholmissbrauch ohne anatomischen Befund ist bisher wenig bekanat gewesen und ganz besonders gilt dies für Alkohollähmungen. Ich werde im Folgenden noch an einer anderen Beobachtung zeigen, dass rein functionelle Alkohollähmungen vorkommen, welche nicht nur einen pe-

1) H. Eichborst, Neuritis diabetica und ibre Beziehungen zum fehlenden Patellarsehnenreflex. Dieses Archiv Bd. 127. S. 1. 1892. 
ripherischen, sondern einen cerebralen Charakter darbieten. Ans der im Vorhergehenden mitgetheilten Beobachtung ersieht man jedenfalls:

1) dass unter dem Einfuss des Alkoholes schwere Veränderungen an den Muskeln bei Unverfersertheit der Nerven zu Stande kommen könuen,

2) dass mitunter alkoholische Lähmungen mit peripherischem Charakter einen apoplektischen Anfang zeigen,

3) dass derartige Lähmungen ohne anatomische Veränderungen bestehen und rein functioneller Natur sein können.

Nicht nur peripherische, sondern auch centrale Lähmungen konnen durch Alkoholismus hervorgerufen und rein functioneller Natur sein. Auch ihnen kann ein apoplektischer Charakter zukommen. Eine solche Erfahrung machte ich im Verlaufe des verflossenen Jahres bei einem 48jährigen Maurer, welcher mit den Erscheinungen einer linksseitigen cerebralen Hemiplegie auf die Züricher Klinik aufgenommen wurde. Der Mann war seit Jahren ein unter seinen Bekannten äbel berächtigter Trunkenbold. Ás 27. December 1891 erlitt der Pat. einen Schlaganfall, wel. cher ron einer linksseitigen Lähmung von Arm und Bein und auch der linken Gesichtshälfte gefolgt war. Der Kranke wurde an 28. December auf die Klinik aufgenommen und verstarb am 3. Januar 1892. Er war stets benormmen und ging unter zunehmendem Collaps zu Grunde. Die makroskopische und mikroskopische Untersuchung von Hirn, Rückenmark and peripherischen Nerven ergab nicht die geringste Veränderung oder Aufalligkeit.

Es mögen die Details dieser immerhin seltenen Beobachtung folgen:

\section{Beobachtung 2 .}

Jacob Frei, Maurer, 48 Jahre alt, aus Zürich, wird am 28. December 1891 auf die Mediciniscbe Klinik aufgenommen. Kr ist so benommen und verwirrt, dass es unmöglich ist, anamnestische Angaben von ibm zu erbeber. Was man von seiner Umgebung erfährt, läuft im Wesentlichen darauf hinaus, dass Patient, seit Jahren ders Schnapstrinken in allerhöebsien Grade ergeben gewesen ist, dass er sich aber trotzdem einer guten Gesundheit zu erfreuen hatte. Am 27. December 1891 habe der Kranke plötzlich das Bewusstsein verloren und sei niedergestürzt, und bald babe man bemerkt, dass er in dem Gẹbrauche der linksseitigen Extremitäten behindert sei. 
Status praesens 29. December 1891. Morgens Temp. $36,5^{\circ}$, Puls 84. Mittags - $\quad 36,5^{0}, \quad-112$. Abends - $36,0^{\circ}, \quad-100$.

Ziemlich kleiner, gracil gebauter Mann mit guter Musculatur und mittelmässigem Fettpolster. Haut trocken, glatt, ohne besondere Exantheme und Verfärbungen.

Patient nimmt eine etwas collabirte Rückenlage ein, wobei er ein wenig nach links bern̈bergeneigt ist. Er liegt mit halb geschlossenen Augen da und ist nicht bei klarem Bewusstsein. Er weiss nicht, wie alt er ist, wieviel $2 \times 3$ ist u. Aebnl., giebt überbaupt nur Antworten, wenn man ihn laut anruft und aufrüttelt und besinnt sich dabei sehr lange. Vorgesprochene Worte spricht er richtig und ohne merkliche Articulationsstörungen nach; auch benenut er mehrfach vorgehaltene Gegenstände richtig. Subjective Beschwerden gieht er nicht an.

Die Temperatur der Haut erscheint nicht erhöht. Der Puls ist regelmässig, klein, ziemlich schwer zu unterdrücken. Athmung oberflächlich und ruhig, auch regelmässig. Keine Oedeme.

Der Kopf ist nach allen Richtungen hin frei beweglich und beim Beklopfen nirgends empfindlich. Gesichtsfarbe eher blass. Skleren rein. Conjunctiven leicht injicirt. Die Pupillen sind ziemlich weit, beiderseits gleich und reagiren bei Lichteinfall, wenn auch etwas träge.

Der Gesichtsausdruck hat etwas Maskenartiges und Steifes und es gewinnt ab und zu den Anschein, als ob eine Schwäche in der linksseitigen Wangen - und Lippenmusculatur vorhanden ist. Jedoch verläuft die Mundspalte horizontal; auch wird die Zunge, welche feucht, etwas weisslich belegt ist und nicht zittert, grade herausgestreckt. Die Inspection des weichen Gaumens ist nicht möglich, weil Patient bei dem Versuch, den Mund zu öfnen, mit aller Kraft die Kiefer auf einander beisst.

Während der Kranke das rechte Bein und den rechten Arm frei und kräftig auf Aufforderung bewegt, vermag er das linke Bein nur unter grosser Anstrengung und Verzerrung des Gesichtes etwas emporzuheben. Dabei schwankt das Bein hin und her und macht einen steifen und ungelenken Eindruck. Passiven Bewegungsversuchen vermag es keinen Widerstand zu bieten. Die Sensibilität erscheint nicht wesentlich verändert, doch sind feinere Prüfungen wegen der Benommenbeit des Patienten unmöglich. Der Patellarsehnenreflex ist zwar beiderseits vorhanden, aber linkerseits lebhafter als rechts. Beiderseits wird Fussclonus vermisst.

Den linken Arm vermag der Kranke kaum zu bewegen. Passive Bewegungen dagegen finden etwas Widerstand; auch verziebt dabei der Patient schmerzhaft das Gesicht. Schwere Sensibilitätsstörungen bestehen nicht. Die Sehnenreflexe sind an beiden Armen gleich stark und lebhaft.

An den Brust - und Bauchorganen sind Veränderungen nicht nachweisbar, nur tritt, wenn man das Herz längere Zeit auscultirt, ab und zu eine vorübergehende Unregelmässigkeit in der Herzbewegung auf.

Patient hat gestern Abend ein wenig erbrochen. Der sparsam gelassene 
Hara ist röthlich-gelb, enthält keinen Zucker, aber gams geringe thiweissmengen. Es ist noch kein Stubl eingetreten. Während der Nacht war Patient unrubig.

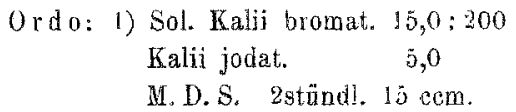

2) Eisblase auf die rechte Kopfseite.

Sraukheitsverlauf.

Der schlaftrunkene und traumbafte Zustand des Kranken dauerte umunterbrochen an und Patient eriagte niemals ein klares Bewrsstsein. Im Schlafe wurden mehrfach leichte Bewegungen an den haken Extremitäten beobachtet, aber die Lähmung blieb deutlich bis zum Tode bestehen. Jer Harn enthielt stets etwas Eiweiss, aber trotz Centrifugirens konnten niemals in ihm Nierencylinder oder andere Formelemente nachgewiesen werden. Gegen das Lebensende bin stellte sich eine unbedeutende Temperaturerhöhung ein. Der Tod trat unter zunehmender Bewusstseinsstörung ein. Ueber das Verbalten der Temperatur, des Pulses, der Harnausscbeidung und des Stuhles giebt nachfolgende Tabelle Aufklärung:

28. Dec. 1891 Abends 6 Uhr T. 36,90, P. 102, Imal Erbrechen.

29. Dec. 1891 Morgens T. $36,5^{6}$, P. 84

$$
\begin{aligned}
& \text { Littags }-36,5^{\circ},-112 \\
& \text { Abends }-36,0^{\circ},-100
\end{aligned}
$$

30. Dec. 1891 Morgens - $36,5^{\circ},-80,300 \mathrm{cem}$ Harn, Harnfarbe Vogel 5, Mittags $\left.-36,4^{0},-78\right\}$ Spee. Gew. 1015 , Eiweiss 0,25 pro Abends - 37,50, - 72 mille, 1 dünner Stubl.

31. Dec. 1891 Morgens - 37,00, - 92 $300 \mathrm{ccm}$ Harn, Harnfarbe Vogel 5 , Mittags - 37,50, - 114 Spec. Gew. 1012, Eiweissspuren, Abends - 37,30, - 120 1 halbfester Stuhl.

1. Jan. 1892 Morgens - 36,30, - 84 | $200 \mathrm{ecm}$ Barn, Harnfarbe Vogel 4, $\left.\begin{array}{l}\text { Mittags }-37,30,-114 \\ \text { Abends }-38,0^{\circ},-120\end{array}\right\} \begin{aligned} & 200 \text { ecm Barn, Harnfarbe Vogel 4, } \\ & \text { Spec. Gew. 1013, Eiweissspuren. }\end{aligned}$

2. Jan. 1892 Worgens - $37,8^{\circ},-96$

Mittags - 37,90, - 138 Secessus nescii.

Abends - $38,0^{\circ},-120$

3. Jan. 1892 Morgens 6 Uhr T. 38, $3^{\circ}$. Tod 7 Uhr Morgens.

Sectionsbefund 3. Januar 1892 Morgens $10 \frac{1}{2}$ Uhr. Männliche Leiche. Auf den unteren Fxtremitäten zahlreiche blaurothe Todtenflecke.

Schädelnähte gut entwickelt. Diploe der Schädelknochen erhalten.

Dura mater gespannt. Im Sinus longitudinalis flässiges Blut und Cruor. Inuenfläche der Dura glänzend, feucht und glatt.

In den Mascben der Pia mässige Mengen klaren Fluidums. Pia nicht besonders getrübt.

In den Sinus der Basis des Schädels reichliche Cruormassen neben flüssigem Blut.

Seitenventrikel des Gehirns nicht erweitert. Linke Grosshirnhemisphäre 
von guter Consistenz und normaler Farbe, mit der gewöhnlichen Zahl von Blutpunkten. Die rechte Grosshirnbälfte ron der gleichen Bescbaffenheit. Dritter Ventrikel etwas weit. An den basalen Grosshirnganglien weder links noch rechts etwas Besonderes.

Substanz des Kleinhirnes feuchter und schlaffer als diejenige des Grossbirnes, aber sonst obne Abnormität.

Arterien der Hirnbasis zart, nur die Carotiden klaffen. Nirgends Verstopfungen in ibnen.

Pons and Medulla oblongata bieten nichts Besonderes dar.

Es wird ein Stäck vom linken Radialnerven herausgeschnitten; makroskopisch nichts Auffälliges an ibm.

Im Duralsack des Rückenmarkes nur sparsame Cerebrospinalflüssigkeit. Auf der hinteren Fläche des Rückenmarkes sind die Gefässe nur schwach gefüllt, nur im Lenden- und Brusttheil erscheinen die Venen stärker gefülit. Das Rückenmark ist von guter Consistenz und zeigt auf Durcbschnitten überall die gewöhnliche Farbe und Zeichnung. Es bietet nirgends etwas Besonderes und makroskopisch keine Veränderungen dar.

Fettpolster nicht übermässig reichlich. Musculatur von guter Farbe und Beschaffenheit. Lage der Baucheingeweide die gewöhnlicbe. Zwerchfellsstand rechts vom oberen Rande der sechsten, links vorn unteren Rande der fünften Rippe.

Die Langen retrabiren sich kaum und berühren sich in der Mittellinie. Pleurahöhle rechts fast leer; rechte Lunge frei. Linke Lunge ausgedehnt verwachsen.

Herzbeutel stark nach links herüber verscboben; er entbält kaum 5 ccm einer wässerigen klaren Flüssigkeit. Mitralostium kaum für 2, Tricuspidalostium für 3 Finger durchgängig. Ueber dem rechten Ventrikel ein Fünffankstück-grosser Sehnenfleck. Subepicardiales Fettgewebe nicht vermehrt. Rechter Ventrikel von gewönnlicher Weite. Pulmonalklappen zart. Consistenz der Musculatur gut. Farbe blassbraunroth. Tricuspidalis zart. Im recbten Atrium viel Cruormassen. Keine Verdickungen des Endocards. Musculatur des linken Ventrikels von guter Consistenz; ihre Farbe etwas blass. Höhle nicht erweitert. Aortenklappen unverändert. Freier Rand der Mitralis leicht verdickt und auf den Segeln der Klappe unbedeutende Fettplaques. Am Vorhof nichts Besonderes. Coronararterien unverändert.

Linke Lunge überall lufthaltig; an der Spitze leicht eingesunken; von mittlerem Melaningebalt. Auf Druck entleert sich wenig schanmige Flüssigkeit. Bronchialschleimhaut leicht geröthet, mit wenig schaumig-schleimigem Inhalt.

Rechte Lunge eher gebläht; eine Einziehung nahe der Spitze. Die Lunge ist vierlappig, indem der rechte Unterlappen durch eine liefe Furche in zwei Lappen getheilt erscheint. Die Lunge ist überall lufthaltig und von derselben Beschaffenheit wie die linke.

Auf dem Zungengrund ein dunkelgrauer Belag. Zungenpapillen gut entwickelt.

Am Pharynx nichts Auffälliges. 
Oesophagusschleimbant nit scomierigen gelblichen Wasson bolackt, die unteren Abschnitte won deutlich galliger Farbe.

Schleimbaut der Trachea ebenfalls mit schleimigen zelblichen Massen bedeckt.

Keblkopf unveränderi. Schilddrüse hleinapfelgross, mit kleinen Colloidknoten, namentlich rechts.

Aorta und Carotis unverändert.

Milz nicht vergrössert, von sehr weicher Consistenz and obne Follikelzeichnung. Milzkapsel an einzelnen Stellen verdickt.

Linker Ureter von gewöhnlicher Weite.

Linke Nebenniere gross und auf dem Durchschnitt von Blutmassen durchsetzt.

Fettkapsel der linken Niere nicht n̈bermässig entwickelt. Die Kapsel !ösi sich leicht. Nierenoberfläche glatt und glänzend. Auf dem Durchschnitt nicbts Auffäliges.

Rechte Nebenniere unverändert, aber auch etwas gross.

Auf der Oberfläche der reckten Niere eine kirschkerngrosse Oyste; sonst die Niere wie die linke.

Dünndarw überall mässig injicirt. Dickdarm weniger injieirt, normal.

Leber nicht vergrössert. Oberfläche glatt und mit einzelnen weissen Flecken durchsetat, die auf dem Durchschnitt bis $0,5 \mathrm{~mm}$ in das Parenchym hineinreichen. Die Leber bietet die gewöbnliche Zeichnung dar.

Pankreas, Blase und Prostata ohne Veränderung.

Anatomische Diagnose. Mässige Erweiterung des dritten Hirnventrikels. Blutung in die linke Nebenniere.

Theile des Gehirnes, Rückenmarkes und des linken Radialnerven wurden theils frisch, theils nach vorhergegangener Behandlung mit Ueberosmiumsäure untersucht, aber an keiner Stelle trat eine Abnormität und Auffälligkeit zu Tage. Bei der Untersuchung der linken Nebenniere zeigte sich das Gebilde mit frischem ausgetretenen Blute durchsetzt. Offenbar handelt es sich hier um einen seltenen Befund, deun bekanntlich stellen Nebennierenblutungen, namentlich bei Erwachsenen kein häufiges Ereigniss dar. Könnte vielleicht diese Blutung dem ganzen Symptomencomplexe zu Grunde gelegen haben? Nach Alledem, was bisher über die Symptome einer Nebennierenblutung bekannt geworden ist, darf man nach unserem Dafürhalten die gestellte Frage getrost mit nein! beantworten. Schmerzen in der Nieren- und Nebennierengegend, fühlbare Resistenzen bei grösseren Blutungen, hochgradige Anämie und Verblutung, wenn das Blut in die Bauchhöhle durchbrach, Erscheinungen von Bauchfellentzändung und hochgradiger Athemnoth, - das sind 
Dinge, welche man bei Nebennierenblutung beobachtet hat, aber nicht apoplektische und hemiplektische Zustände. Zudem war die Nebennierenblutung bei unserem Patienten keine sonderlich umfangreiche und liegt es viel näher, sie als eine Blutung aufzufassen, welche von der Hemiplegie abhängig ist. Ist es doch bekannt, dass in paarigen Organen auf der gelähmten Seite gar nicht zu selten Blutungen bei Hemiplegischen angetroffen werden, deren Entstehung man vielfach auf vasomotorische Störungen zurückgeführt hat. Und so glauben wir ein Recht zu haben, die mitgetheilte Beobachtung als eine alkoholische functionelle Hemiplegie auffassen zu dürfen.

Begreiflicherweise geben die im Vorhergehenden mitgetheilten beiden Beobachtungen noch keineswegs eine Berechtigung für die Annahme ab, als ob nun alle Alkohollähmungen mit apoplektischem Charakter auf rein functionelle Störungen zurückzuführen wären, und gerade im Gegensatz zu den beiden vorausgehenden Erfahrungen wird es vielleicht nicht ohne Interesse sein, eine dritte Beobachtung kennen zu lernen, in welcher urplötzlich zunächst eine Lähmung beider Beine auftrat, so dass man an einen Sitz der Krankheit im Rückenmarke hätte denken können, bei der es aber nicht zweifelhaft sein konnte, dass eine. apoplektische Polyneuritis acuta vorlag.

Die Beobachtung betrifft eine 34jährige Kellnerin, welche in den letzten Jahren einem Bordelle vorstand, und in dieser Stellung fast täglich in Alkoholgenuss excedirte und sehr häufig auch geschlechtliche Ausschweifungen durchmachte. Von Geschlechtskrankheiten blieb sie trotzdem verschont. Auf einer Reise stïrzt sie auf einem Bahnhof zusammen und ist an beiden Beinen gelähmt. Man verbringt sie nach 8 Tagen auf die Züricher Klinik. Hier stellt man eine schlaffe Lähmung der Beine fest; die gelähmten Muskeln magern schnell ab, zeigen elektrische Entartungsreaction und sind gleich den Nerven auf Druck sehr empfindlich. Ausserdem klagt die Patientin über die heftigsten Schmerzen in den gelähmten Beinen. Dabei besteht Anästhesie der Haut. Die Patellarreflexe sind erloschen. Blase und Mastdarm functioniren normal.

Ungefähr zwei Wochen später stellen sich Parästhesien in beiden Armen und bald darauf linksseitige Radialislähmung ein. 
Die Lähmungen gehen orst nach länger als oinem halben Jahr langsam zurück. In der Wadenmusculatur bildet sich daneben eine sehr starke Contractur aus, so dass Patientin auf der chirurgischen Klinik tenotomirt ist. Nach diesem Eingriffe lernt sie zunäcbst mit Krücken allein gehen; nach einiger Zeit bedarf sie aber der Krücken nicht mehr und kann geheilt entlassen werden.

Im Anschluss an das in aller Kürze Mitgetheilte saöge die Krankengeschichte, welche zeigt, wie bei genügender Geduld und Ausdauer auch sehr schwere Alkohollähmungen zur Heilung fühsen können, genauer und ausfübrlicher folgen.

\section{Beabachtung 3 .}

Emilie Imholz, 33 Jahre alt, Kellnerin, wurde am 11. April 1889 auf die medicinische Klinik in Ztirich aufgenommen und stand hier bis zum 3. Tuvi 1890 in fast ununterbrochener Beobachtung und Behandlung.

Anamnese. Der Vater der Patientin verstarb vor kurzer Zeit in Folge eines Herz- und Nierenleidens, die Mutter an Magenkrebs.

Von ibren 14 Geschwistern gingen 9 vor Vollendung des ersten Lebensjabres an "Gichtern" 20 Grunde, die 5 übrigen sollen alle gesund sein.

Patientin will mehrfach Kinderlsankheiten durchgemacht baben, ohne jedoch die einzelnen Krankbeiten benennen zu können. Sie wurde im 13. Lebensjabre zum ersten Male menstruirt; die Menses stellten sicb nicht selten zu früh ein. Seit dieser Zeit machte sich Neigung zur Fet leleibigkeit bemerkbar. Im 18. Lebensjahre erlitt die Patientin einen Fall auf die Stirn. Sie war danach einige Zeit bewnstlos und litt vielfach Monate lang an Kopfschmerzen. Auch wurden inr an einer umschriebenen Stelle über dem linken Scheitelbein die Haare grau.

Patientin ist seit 10 Jahren Kellnerin. Sie gesteht zu, in dieser Stellung sehr viel Bier, Wein und auch Schnaps getrunken zu haben. Seit über 2 Jahren übernahm sie die Teitung eines Bordelles. Die alkoholischen Ausschweifungen nahmen dabei nicht ab und gesellten sich noch häufige geschlechtliche Ausschreitungen hinzu. Patientin blieb aber von Geschlechtskrankheiten versehont.

Seit etwas länger als einem halben Jabre stellten sich nicht selten reissende Schmerzen, namentlich in der Wadenmusculatur ein, gegen welche nicht obne Erfolg spirituöse Finreibungen versucht wurden. Die letzten vier Monate hielt sich die Kranke in München auf. Hier erkrankte sie unter Magenbeschwerden und erbrach sehr bäufig; namentlich am Morgen. Die Erscheinungen verschlimmerten sich vornehmlich nach Biergenuss, so dass die Patientin sich an tägliche grössero Mengen Kräuterliqueurs hielt.

Am 5. April 1889 trat Patientin eine Geschäftsreise von München nach Biel an. Von München bis Lindau batte sie grösstentbeils tief und gut 
geschlafen. Als sio in Lindau den Eisenbahnwagen verlassen batte, um sich auf das Dampfschiff zu begeben, stürzte sie plötzlich auf dem Perron znsammen, weil ibr die Beine gelähnt waren. Schmerzen hatte sie dabei nicht, doch machte sich nach einiger Zeit ein Gefühl von Fingeschlafensein beuerkbar. Sie wurde vom Dienstpersonal zunächst auf das Dampfsehiff und nach beendeter Ueberfahrt in Rorschach in den Eisenbahnwagen getragen. In Zürich empfingen sie Verwandte, bei welchen sie Unterkunft fand. Da sie in den nächsten Tagen, wie auch schon in München, mehrfach brach, soll der hinzugezogene Arzt sie zunächst nur wegen Magenkatarrhes behandelt und der Läbmung ibrer Beine erst dann Aufmerksamkeit geschenkt baben, als hier unerträgliche Schmerzen auftraten. Am 11. April 1889 wurde sie mit der Diagnose eines Magenkatarrbes und wahrscheinlichen Rüchenmarksleidens auf die Züricher Klinik verbracht.

Status praesens 12. April 1889. Grosse Person von sebr bedeutender Fettleibigkeit. Gesichtsfarbe roth. Ausgedebnte Acne rosacea auf Nase, Wangen und Kinn. Blick unruhig und unsicher.

Patientin ist bei freiem Sensorium und klagt über Lähmung und Schmerzen in den Beinen. Die Schmerzen bestünden zwar unterbrochen, steigerten sich aber anfallsweise bis zur Unerträglicbkeit und breiteten sicb vornebmlich auf der vorderen und inneren Fläche der Ober- und Unterschenkel aus.

Patientin liegt auf dem Rücken und hält diese Lage meist inne, da ibr Seitenlage wegen der Lähmung der Beine unbequem sei.

Die Temperatur fühlt sich nicht erböht an. Die Temperatur der Achselböhle betrug heute am Morgen $37,4^{\circ} \mathrm{C}$, gestern Abend nach der Aufnahme $38.2^{\circ} \mathrm{C}$. Der Puls ist regelmässig, leicht unterdrückbar, ein wenig beschleunigt, 100 Schläge in der Minute. Keine Dyspnoe. Keine Oedeme.

Gesichtsausdruck etwas ängstlich. Skleren rein. Pupillen eng, aber auf Lichtreiz lebhaft und beiderseits gleich gut reagirend. Zunge feucht und rein. Keine Lähmungen oder Anästhesien im Gesicht. Schädel beim Beklopfen nirgends empfindlicb. Kopf frei beweglicb.

An der Wirbelsäule keine Deformitäten und keine Druckpunkte.

An den Armen und Händen keine Veränderung nachweisbar.

Die unteren Extremitäten sind vollkommen gelähmt und Patientin vermag nicht die leiseste Bewegung auszuführen. Die Lähmung ist eine vollkommen schlaffe. Wenn man den Versuch macht, die Kranke auf die Beine zu stellen, so sinkt sie in sich zusammen. Druck auf die gelähmten Muskeln und Nerven ruft aufällig lebhaften Scbmerz bervor. Die geläbroten Beine zeigen normale Farbe und Temperatur. Es bestebt vollkommene Anästhesie der Haut, so dass auch sehr tiefe Nadelstiche der Haut nicht wabrgenommen werden. Temperatur- und Muskelsinn erscheinen unverändert. Die Patellareflexe fehlen. Blasen- und Mastdarmthätigkeit ungestört.

An den Brust- und Baucborganen nicbts Abnormes.

Kein Auswurf. In den letzten 12 Stunden wurden $500 \mathrm{ccm}$ hellgelben Harnes entleert. Der Harn entbalt weder Wiweiss noch Zucizer und besitzt ein specifisches Gewicht von 1015. Ein fester Stuhl. 
Parientin hat keinen Appetin, aber gesteigerten Durst und Klagt nber gestörten Schla?.

Diagnose. Apoplektische Altoholneuritis der Beine.

Ordo. Sol. Kalii jodati 5,0:200. DS. $3 \mathrm{mal}$ täglich $15 \mathrm{ecm}$.

Krankbeitsverlaut.

25. April 1889. Patientin war stets fieberfrei und ihre dchselböhlentemperaturen schwankten 7 wischen $36,1-37,4{ }^{\circ}$. Der Puls blieb beschleuaigt (bis 124), war aber immer regelmässig. Es wird noch immer über die heftigsten Schmerzen in den Reinen geklagt. Die gelährnten Muskeln sind sehr stark abgemagert. Der Musculus tibialis anticus und Extensor digitorum communis reagiren auf den faradischen Strom gar nicht. Bei Prüfung mit dem galvanischen Stror beobachtet $\operatorname{man~KaSZ}=18 \mathrm{M}$. A. und $\mathrm{AOZ}=14 \mathrm{M}$. A. Durch das starke Fettpolster ist die Prüfung sebr erschwert.

Seit der Nacht klagt die Patientin über taubes Gefünl in beiden Händen and über Schwäche in Armen und Händen. Sie kann alle Bewegungen ausfübren, doch sind dieselben sehr schwach. Bei Prüfungen mit der Nadel sind Sensibilitätsveränderungen nicht nachweisbar.

29. April 1889. Bei andauernd fieberfreiem Zustand bat sich eine ansgesprochene linksseitige Radialisläbmung entwickelt. Die Sensibilitäł der Haut erscheint erhalten, nur die Iteitung verlangsamt. Patientin wird noch iwmer durch sebr starke Schwerzen in den Armen und Beinen belästigt.

Ordo. Acid. salicylic. 1,0. $3 \mathrm{mal}$ täglich 1 Pulver.

3. Mai 1889. Seit vier Tagen wird die Kranke wăhrend der Nacht sebr autgeregt und kann keinen Schlaf finden. Dabei ist ihr Bewusstsein nicht vollkommen klar. Sie hallucinirt mehr und mehr, sieht nawentich oft Näuse, Tauben, Käfer und wacht den Eindruck eines Delirium tremens.

Ordo. Sulfonal 2,0. Am Abend 1 Pulver.

24. Mai 1889. Delirien und Schlaflosigkeit baben bis vor 3 Tagen fortgedauert. Der Zustand änderte sich unverkennbar, als dje Patientin seit dem 18. Mai täglich 200 Cognae erhielt. Zugleich bürte das Erbrechen aut, welches täglich mehrmals sich einzustellen pflegte. Patientin hatte Abends häufig Temperaturerhebungen (bis zu $39,2^{\circ}$, in der Regel freilich nicht über $\left.38,2^{\circ}\right)$. Seit dem 20. Mai ist sie wieder vollkommen fieberfrei.

Gegen die sehr lästigen Scbmerzen in den Armen und Beinen erbieft die Kranke täglich Morphium $(0,01)$ subcutan. Seit. 8 Tagen sind die Sehmerzen vollkommen verschwunden und treten nur noch bei sehr kräftigem Druck auf die gelähmten Nerven und Muskeln auf. Zugleich jst wehr und mehr die Hautsensibilität in den Beinen wiedergekebrt, so dass man gegenwärtig keine Störungen mehr nachweisen kann.

Ordo: Sol. Kalii jodat. 5,0:200 3mal täglich 1 Esslöffel.

31. Mai, Linker Radialnerv und lintes Bein sind noch jumer vollLommen gelahmt, dagegen ist die Patientin jetzt im Stande, das rechte Bein in Hüft- und Knjegelenk $z u$ beugen. Freilich vermag sie es uicht von der Bettunterlage emporzubeben; auch kann sie keine Bewegungen wit den Zehen und in dem Fussgelenk ausfübren. 
10. August. Seit einigen Tagen ist die Patientin im Stande mit der linksseitigen Radialismusculatur Bewegungen zu machen. Die Kraft iśt reilich sebr unbedeutend.

16. August. Die Kranke vermag jetzt auch das linke Bein im Hüftund Kniegelenk zu beugen. Sie hebt jetzt beide Füsse bis $16 \mathrm{~cm}$ von der Bettunterlage empor. Patellarsehnen- und linksseitiger Tricepssehnenreflex fehlen noch immer vollständig.

Ordo: Massage und Faradisation.

14. Sept. Seit dem Spitaleintritt haben sich zum ersten Male die Menses eingestellt.

28. Septu In den letzten Tagen sind wieder boftige Schmerzen in den Beinen aufgetreten, die pamentlich gegen Abend eintreten.

$$
\begin{aligned}
& \text { Ordo: 1) Aatipyria } 0,5 \text { - 2stündl. } \\
& \text { 2) Massage und Faradisation ausgesetzt. }
\end{aligned}
$$

3. Oct. Die Schmerzen in den Beinen sind geringer geworden. Die linksseitige Radialisläbmung ist ganz geschwunden. Patientin hebt die Beine mehr als einen halben Meter hoch, bcugt und streckt sie kräftig, nur kann sie noch immer keine Bewegungen mit den Zehen und in den Fussgelenken machen. Die Füsse nehmen mehr und mear eine Varo-equinusStellung ein.

6. Dec. Patientin gebraucht ihre Arme wie in gesunden Tagen. In den Beinen treten immer noch ab und zu Scbmerzen auf. Die Beuger der Ober- und Unterschenkel haben ibre Function und Kraft wiedergewonnen, dagegen besteht noch beiderseits vollkommene Peronenslähmnng. Die Wadenmusculatur ist in sebr starke Contractur geratben und die Pes-Varo-equinusstellung hochgradiger und unbeweglicher denn jemals zuvor. Der Patellarsehnenreflex fehlt noch jmmer.

24. Februar 1890. Der Zustand der Patientin hat sich in keiner Weise geändert, nur bat die früher atrophische Musculatur der Oberscbenkel wieder einen normalen Umfang angenommen. Gebversuche scheitern an der Spitzfussstellung. Es wird daber die Patientin auf die chirurgische Klinik gelegt, um an ibr die Tenotomie der Achillessehne vorzunehmen.

Patientin befand sich vom 24. Februar bis zum 26. April 1890 auf der chirurgischen Klinik. Aus dem Krankenjournal der letzteren, welches mir mein College Krön]ein in zuvorkommendster Weise zur Verfügung gestellt bat, entnebme ich Folgendes:

Patientin wutde am 28. Februar 1890 in Chloroformuarkose, weiche sehi turbulent verlief, tenotomirt. Man gab den Füssen eine Stellung im rechten Winkel zum Unterscbenkel und zugleich eine Valgusstellung und legte sie in einen Gypsverband. Am 27. März wurde der Gypsverband entfernt, wonach beide Füsse in normaler Stellung verblieben. Am 30. Märn machte die Kranke mit Hülfe von Krücken die ersten Gebversuche. Wegen des sehr bedeutenden Körpergewichtes fielen dieselben zmnächst sehr mangelhaft aus, allein alltählich konnte die Patientin durch tägliche Uebungen besser und

Archiv f. pathol. Anat. Ba. 129 . Het. 1. 
besser gehen und wird an 26. April auf die medicinische Rlinik zurüctsverlegt.

Patientin blieb auf der medicinischen Klinik pom 26. April bis zum 3. Juni 1890. Es bestand noch immer vollkommene Peroneuslähmung; auch war die Extensorenmusculatur der Unterschenkel stark abgemagert. Es wurde tägliche Massage und Faradisation der gelähmten Muskeln verordnet.

4. Mai. Patientin bewegt die Zehen und kann die Füsse dorsalwärts flectiren. Sie geht sicherer, ermädet weniger und stösst nicht mehr so oft beim Geben mit den Zehenspitzen auf den Fussboden auf.

27. Mai. Patientin geht ohne Krücken und wird nur bei Ermüdung etwas wackelig und zitternd.

3. Juni. Patientin wird geheilt entlassen. Patellarsehnenreflexe fehlen noch immer, doch sind die Läbmungen sämmtlich verschwunden.

\section{Erklärung der Abbildungen. \\ Taî̉ IV.}

Wig. 1. Querschnitt aus dem Musculus extensor digitorum communis des rechten Unterarmes. Hämatoxylin-Eosinpräparat. Vergr. 37 fach.

Fig. 2. Dasselbe bei 280facher Vergrösserung. 